\title{
Marxism and Nationalism in the Albanian Historiography School: The Position of the National Hero
}

\author{
Doan Dani \\ Dottore in Scienze Storiche e Documentarie \\ doandani@gmail.com
}

\section{Doi:10.5901/mjss.2014.v5n6p251}

\begin{abstract}
The present article examines the influence of the ideological approaches as Marxism and Nationalism in the Albanian historical school of Middle Age after the Second World War. This two perspectives of historical interpretation have generated some specific contradictions, particularly about Scanderbeg's authority, the Albanian's national hero: it seems to be both 'feudal lord' and 'peasant patriot' who fight for principles as Nation and Europe, very abstract in fifteenth century. The paper traces the construction of Scanderbeg's authority by some of the most important Albanian historians.
\end{abstract}

Keywords: Marxism, Nationalism, Middle Age, Feudalism.

\section{The Ideological Dualism (II Dualismo Ideologico)}

In linea massima la storia medievale albanese è stata modellata da due approcci fondamentali: il nazionalismo e il marxismo. Essendo una scuola recente, poiché sviluppata sostanzialmente nel Secondo dopo guerra, l'approccio nazionalistico e andato ridimensionandosi in favore a un interpretazione di stampo patriottico, meglio compatibile al regime comunista instaurato dopo il 1944. Ciononostante la dimensione funzionale di questa storiografia rimane predominante: essa deve servire al regime, alla patria, al popolo, e di conseguenza il passato viene modellato in base alle esigenze ideologiche del momento. Ciò che interessa a quest'analisi è la fusione della prospettiva storiografica marxista e quella nazionalista, impostata come modello interpretativo dominante dei rapporti sociali medievali, della saga epica dell'eroe nazionale albanese Scanderbeg (1405-1468), del feudalesimo e dell'Altro in generale.

Non solo feudalesimo è una creazione moderna offerta a partire da Montesquieu in poi, come giustamente osserva Chris Wickham (2000: 12), ma anche lo stesso oggetto degli studi legato al concetto è stato spesso costruito dall'uomo moderno. Dalla prospettiva marxista «l'idea feudale ne risulta spostata dal piano giuridico-militare al piano economicosociale e per feudalesimo si intende lo sfruttamento politico-economico dei contadini» (Sergi, 2005: 45), ed è proprio questa tipologia dei rapporti verticali - sintetizzata nella conflittualità - che genera identità per l'albanese medievale visto dall'omonima scuola storiografia, nella quale il feudalesimo si sovrappone al medioevo e finisce addirittura per configurarlo. La pienezza del quadro interpretativo dei rapporti medievali si raggiunge associando alla conflittualità verticale la componente nazionalistica che vede il contadino medievale albanese impegnato nella lotta perenne contro il dominio straniero. Non a caso nella vulgata albanese del 1967 la parola insurrezione - nelle pagine riguardanti l'entrata degli albanesi nella storiografia (metà XI secolo) fino all'inizio dell'impatto con gli ottomani (fine Trecento) che è esclusivamente impregnato dalla conflittualità - per la sua frequenza non è seconda a nessun'altra parola del lessico conflittuale (Frashëri, Islami, 1967: 169-235) ${ }^{1}$. In più, l'esclusività dell'uso di questo termine di fronte ad altri - ribellione, tumulto, sommossa, ammutinamento ecc. - è tutt'altro che casuale, in quanto rende meglio l'idea dell'impegno massiccio dei ceti inferiori («la massa contadina») insieme alla resistenza e alla libertà in veste archetipica. II linguaggio usato per la descrizione delle insurrezioni registrate nell'Albania medievale è strettamente improntato dal contrasto fra massa popolare compatta e classe degli sfruttatori, identificati come stranieri o semplicemente come signori feudali. Si fa strada così l'idea di un antifeudalesimo insito nel popolo albanese: lo sfruttamento signorile e la reazione contadina - due linee principali della medievistica di carattere marxista delineata da Wickham (2007: 37) - permeano l'intera medievistica albanese.

\footnotetext{
1 Il termine ritorna per più di sessanta volte, mentre a dominio si ricorre cinquanta volte, guerra/battaglia trentacinque volte, sfruttamento diciannove volte, giogo tre volte, e, per fare un paragone, cultura otto volte, popolo quattordici volte, massa (popolare o contadina) venticinque volte.
} 


\section{The Ideological Contradictions (Le Contraddizioni Ideologiche)}

Tuttavia, l'equazione del conflitto 'contadini-signori più popolo-stranieri' genera una situazione paradossale a dir poco confusa e conosce un suo limite quando si deve affrontare il messianismo dell'eroe nazionale Scanderbeg, il quale allo stesso tempo era anche un signore, e quindi teoricamente sfruttatore dei contadini suoi "connazionali". L'approccio patriottico-marxista tende verso la snaturalizzazione della sua autorità signorile nella prospettiva di una simbiosi tra eroe e massa, in specifico contadina. Tale prospettiva raggiunge la massima espressione nell'opera di Fan Noli che si è contraddistinta innanzitutto per una terminologia tipicamente marxista: espressioni come «imperialismo albanese», «lotte di classi», «sfruttatori e sfruttati», «borghesia», «proletariato», «liberi contadini», «servi», «proprietari [çifligar]», «giogo straniero», «eserciti feudali» (Noli, 1921: 34-36; 1950: 9-27; 8-44, 150-153), costellano le due opere a tal punto da attirare, nella seconda edizione pubblicata negli anni Quaranta, anche le critiche dello storico Aleks Buda (1951: 139-164; 2000: 82-112). La suddivisione tra «sfruttatori» e «sfruttati», tra «signori feudali» e «servi della gleba» - scaturita dalla concezione marxista - viene applicata da Noli soltanto alla realtà medievale dei territori conquistati da Venezia e dall'impero ottomano, mentre laddove si estende l'autorità dei signori albanesi simili distinzioni scompaiono. II suo Scanderbeg appartiene a una categoria da lui battezzata come «aristocrazia patriarcale», caratterizzata dalla mancanza di enormi proprietà fondiarie e dalla vicinanza con la massa contadina: un ceto che, secondo lo storico, si collocherebbe tra «l'aristocrazia», «la borghesia» e «il proletariato» (Noli, 1989: 344-346). Date queste premesse diviene impossibile trovare tracce di sfruttamento dei contadini da parte dell'eroe nazionale o degli altri signori albanesi impegnati nelle insurrezioni. I contadini appaiono in uno stato libero, scelgono volontariamente di arruolarsi nell'esercito o di condividere le sorti della guerra: quindi non è possibile comprende il loro rapporto giuridico ed economico con i signori che detengono l'autorità, anzi, di fronte a tale appiattimento delle differenze economiche, sociali, politiche e giuridiche, i rapporti verticali si dissolvono in quelli orizzontali. Noli tende a sottolineare che gli «sfruttatori» della «massa» sono sempre gli «occupanti stranieri» ${ }^{2}$. Scanderbeg invece è figlio del popolo, un plebeo che si sacrifica per ideali sublimi, il Salvatore della nazione e della civiltà europea, il modello della società senza classi, insomma, un "rivoluzionario nel medioevo".

In apparenza gli storici albanesi non potevano accettare le posizioni di Noli per il semplice motivo che, in assenza di rapporti feudali, l'Albania sarebbe risultata troppo arcaica; in mancanza di ciò anche la tesi che attribuisce a Scanderbeg il superamento di questa fase sarebbe risultata improponibile, ma, inoltre, anche la tesi che vedeva nell'attività di Scanderbeg un azione proiettata verso la nascita della monarchia, ovvero dello stato albanese medievale (Buda, 1951: 154-155), ne sarebbe stata compromessa. Secondo Buda soltanto l'appartenenza di Scanderbeg alla classe dominante poteva creare le condizioni per realizzare questi progetti: la mobilitazione generale in guerra, le alleanze e il superamento del separatismo (Buda, 2000: 97-98). Per giunta la critica a Noli, circa di annichilimento delle differenze di classe, appariva scontata. Ciononostante la critica di Buda non concerne l'approccio marxista al medioevo, o meglio, non ci troviamo di fronte a un cambiamento di prospettiva: permane la medesima analisi dei rapporti fra «sfruttatori/feudali» e «sfruttati/contadini», ivi inclusi anche i signori albanesi, che ha come logica conseguenza la tesi della conflittualità perenne fra le classi.

Per avvalorare l'immagine di Scanderbeg-signore feudale Buda si serve di alcuni esempi diacronici e sincronici che attesterebbero lo sviluppo del feudalesimo in Albania, almeno a partire dal Duecento, come appunto comproverebbero l'esistenza dei «servi della gleba» (bujkrobër) e «la conflittualità signorile» (Buda, 1951: 149-156; 1967: 3-14). Gli studi di altri storici - Selami Pulaha, Kasem Biçoku - sulla situazione economica e politica nella prima metà del XV secolo (Pulaha, 1970: 111-157; Biçoku, 1975: 137-150; 1976: 163-183), insieme agli studi delle istituzioni giuridiche e politiche dello stato di Scanderbeg (Frashëri, 1977: 125-138; 1978: 20-21; Luarasi, 1982: 173-182; Hadri, 1985: 55-73), danno per scontata l'appartenenza di Scanderbeg e dei suoi rivali alla classe feudale, trascurando del tutto l'analisi dei rapporti giuridici all'interno dei domini di Scanderbeg e quelli fra i vari signori. Aleks Luarasi, esperto nel campo del diritto, ma non un medievista, quando analizza l'apparato giuridico dell'epoca di Scanderbeg si limita a esaminare i campi del «diritto penale», «canonico» e «consuetudinario». \| primo potrebbe essere interessante per comprendere l'amministrazione della giustizia nei domini di Scanderbeg, ma gli unici casi specifici menzionati riguardano l'accusa di tradimento dei fedeli Moisi Golemi e Hamza Castrioti ${ }^{3}$, la confisca delle proprietà del primo e l'imprigionamento del

\footnotetext{
${ }^{2}$ Anche qua il lessico è significativo. Il termine occupante si sovrappone al più corretto dominante. Questo perché il dominio presume un periodo di lunga convivenza con il conquistatore durante il quale è impossibile ostruire l'osmosi tra «Noi» e l'«Altro», cosa che invece in una visione patriottica della storiografia risulta una contaminazione culturale e quindi nazionale, oltre a erodere le basi teoriche della perenne resistenza archetipica.

${ }^{3}$ Hamza Kastrioti, il nipote di Scanderbeg, secondo la motivazione ormai classica nella storiografia albanese, probabilmente dopo essere stato scavalcato nella linea ereditaria dalla nascita del primogenito di Scanderbeg, si pone al servizio di Maometto II e alla testa di un esercito ottomano tenta di sconfiggere lo zio, ma l'esito della battaglia si conclude con la disfatta di Hamza. Nella vulgata albanese
} 
secondo (Luarasi, 1982: 74 sgg.). Nell'edizione aggiornata e ampliata della propria opera Luarasi si concentra alla situazione giuridica degli strati inferiori, con esempi dispersi e disorganici, senza rilevanti riferimenti al dominio di Scanderbeg. Le fonti di Luarasi rimangono gli studi della prima metà del Novecento, il cosiddetto Kanun (canone) di Scanderbeg ${ }^{4}$, la cronaca aulica e per certi versi fiabesca di Marino Barlezio e la produzione folcloristica (Luarasi, 1998: 117 sgg.). Del rapporto di Scanderbeg con i soggetti presenti nei suoi domini e del suo potere coercitivo non sappiamo praticamente nulla in modo diretto, se non dalla cronaca di Barlezio, dove Scanderbeg appare come un classico primus inter pares che ritorna nelle vesti del Salvatore, che non costringe e non mette tasse, che addirittura è indotto al matrimonio dai suoi sudditi, insomma, una figura piuttosto paterna (Barleti, 2005: 199-200). Barlezio descrive con dialoghi lusinghieri l'incredibile intesa fra il volgo e il suo signore, e a tal punto che questi si configura più come una guida popolare che come un signore, esattamente come aveva ipotizzato Noli a proposito della classe «dell'aristocrazia patriarcale».

Nella critica multidirezionale rivolta a storici come F. Noli, F. Babinger, H. Inalcik, N. Jorga, C. Marinescu e G. Valentini, Buda sintetizza nuovamente la sua posizione sui rapporti di potere nel XV secolo. Secondo lo storico quattro erano gli elementi cruciali della resistenza albanese: la capacità dell'economia albanese di «autorigenerarsi», l'impegno delle «masse popolari» nella resistenza, «la libertà come tradizione» delle «masse popolari» e «la classe signorile» (Buda, 1967: 8-9). Anche il primo fattore è sempre derivante dalle masse che Buda, in un passaggio, definisce perfino come «masse lavoratrici» (Ibidem: 10). Scanderbeg, sostiene Buda, aveva percepito l'importanza politica della massa e dei suoi interessi, ovvero la «terra», la «libertà», l'«unione», l'«indipendenza», la «difesa della cultura europea» (Ibidem: 12). In altri termini, è il popolo a delegare il potere e l'autorità politica a Scanderbeg, che si pone al servizio del popolo piuttosto che dei propri interessi, nonostante appartenga a una classe definita da questi stessi interessi. Egli appare come figlio del popolo posto al suo servizio, diversamente dall'immagine da manuale marxista di un signore medievale, assumendo in questo modo le sembianze del leader comunista Hoxha. II messianismo di Scanderbeg si associa a quello del Partito del Lavoro albanese (partito comunista), che a sua volta si identifica nel leader Hoxha: non può nulla senza la massa; il partito è il popolo; è lo strumento del popolo (Mury, 1971: 161-183). Eppure Buda criticava Noli proprio per non avere visto in Scanderbeg il membro del feudalesimo, anche se poi egli stesso non esitava ad assimilarlo al popolo o, per così dire, a "defeudalizzarlo". Inoltre, veniamo a conoscenza che i contadini medievali combattevano per la «nazione», lo «Stato»e, fatto ancora più inedito, per l'«Europa» ${ }^{5}$.

\section{Conclusion (Conclusioni)}

Nonostante la storiografia albanese del Secondo dopo guerra abbia sostenuto con vigore il carattere feudale dei rapporti fra i ceti sociali dell'Albania medievale, contemporaneamente - almeno nella figura dell'eroe nazionale - si è creata l'immagine di un signore feudale sempre più vicino al popolo, capace di punire i «nemici esterni» e i «traditori», e in grado di porre fine all'«anarchia»: se da un lato l'identità signorile giustifica una serie di componenti dell'equazione interpretativa medievistica albanese, in particolare evidenziando il carattere marxista di tale scuola, dall'altro proprio quest'identità entra in crisi dall'influenza nazionalistica che necessità un signore popolano. Già il termine massa ha un suo significato aggregante e livellatore delle differenze. Nella produzione storiografica albanese non si menziona un solo caso di

il gesto di questo personaggio, fedele a Scanderbeg fino al 1457, viene definito semplicemente «tradimento». L'approccio non sembra evoluto anche nelle recenti riflessioni di Frashëri e Aurel Plasari, nonostante, almeno in un caso per certi versi analogo, quest'ultimo giustifica "l'infedeltà" di Scanderbeg verso Murat II, nel 1443, come azione moralmente corretta e politicamente legittima (Frashëri, Islami, 1967: 292; Frashëri, 2002: 344-345; Plasari: 2010: 821). Biçoku invece non usa la definizione di tradimento o traditore (Biçoku, 2005: 134-136).

${ }^{4}$ Certo è che la carenza di documenti non permette di definire il quadro giuridico dei domini di Scanderbeg, tanto più che in alcuni casi le fonti grazie alle quali gli studiosi albanesi provano a definire lo Stato feudale di Scanderbeg sono estremamente fragili e contraddittorie, come dimostra lo studio del Kanun di Scanderbeg. La prima testimonianza sull'esistenza di norme consuetudinarie elaborate da Scanderbeg risale alla metà dell'Ottocento (Hahn, 1854: 173), ma la raccolta delle norme interessò gli etnologi soltanto nel Secondo dopoguerra (Frashëri, 1977: 125-126.), e l'unica base del Kanun medievale fu la memoria delle generazioni di metà Novecento: è come sostenere con certezza storiografica che Ossian è l'autore autentico dei canti a lui attribuiti. Oltre a essere estremamente friabile come fonte di interpretazioni giuridiche o storiografiche, il Kanun è anche l'insieme di norme tipiche di una società paritaria basata su rapporti fra uomini liberi, dove le decisioni vengono prese in forma collegiale, e con origini che si perdono nei secoli precedenti al feudalesimo. Secondo Frashëri tali normative hanno caratterizzato nel medioevo balcanico quelle comunità che non conoscevano «l'amministrazione statale feudale», con un prolungamento in alcune zone dell'Albania fino all'inizio del Novecento. In vista all'importanza attribuita al Kanun nei rapporti di metà Quattrocento Luarasi contribuisce ad arcaizzare ulteriormente la tipologia dell'autorità attribuita a Scanderbeg, così da creare l'immagine di una società abbastanza livellata e da avvalorare ulteriormente la tesi di Noli.

${ }^{5}$ La domanda di Anthony Smith su «chi morirà per l'Europa» sembra avere già una risposta dal medioevo (Smith 2000: 148). 
conflittualità tra il leader-eroe e i suoi seguaci, perché non ci sono i segni dell'autorità, e non ci sono nemmeno tracce di rapporti materiali, cioè elementi fondamentali quali la tassazione, le corvée, gli obblighi e i doveri, il diritto sulla proprietà, l'esercizio delle prerogative giudiziarie. Quale che fosse l'autorità di Scanderbeg, a fronte dell'immedesimarsi negli ideali e nelle aspettative del popolo, da come viene illustrata essa poteva valere solo come autorità etico-morale. Secondo l'approccio marxista Scanderbeg dovrebbe essere definito un signore feudale, ma paradossalmente, a causa dell'influenza nazionalistica, egli non appare come sfruttatore. In quanto signore feudale avrebbe dovuto essere guidato dai suoi interessi di classe, ma questi coincidevano con quelli del popolo, ovvero, della classe degli sfruttati. Infine, avrebbe dovuto considerare l'anarchia come la norma, ma egli invece la combatteva, quasi come un moderno rivoluzionario inteso a rovesciare tale norma. L'eroe quindi diventa il delegato della massa contadina di fine medioevo, mentre il personaggio storico si trova schiacciato tra il peso marxista sommato e in qualche modo amalgamato con quello nazionalistico.

\section{References}

Barleti, M. (2005), Historia e jetës dhe e veprave të Skënderbeut, $4^{\circ}$ ed., Tiranë: Infbotues.

Biçoku, K. (1975), Rreth pronësisë së tokës dhe strukturës shoqërore të fshatit shqiptar të zonës së Shkodrës në fillim të shek. XV, in «Studime Historike», No. 3, pp. 137-150, Tiranë: Instituti i Historisë pranë Akademisë së Shkencave të Shqipërisë.

Biçoku, K. (1976), Vështrim mbi ekonominë dhe demografinë e fshatit shqiptar të shek. XV, in «Studime Historike», No. 4, pp. 163-183, Tiranë: Instituti i Historisë pranë Akademisë së Shkencave të Shqipërisë.

Biçoku, K. (2005), Skënderbeu dhe Shqipëria në kohën e tij, Tiranë: Botimpex.

Buda, A. (1951), Fytyra e Skënderbeut në dritën e studimeve të reja, in «Buletin i Institutit të Shkencave», No. 3-4, pp. 139-164, Tiranë: Instituti i Historisë dhe Gjuhësisë pranë Universitetit Shtetëror të Tiranës.

Buda, A. (1967), Rreth disa problemeve të historisë të epokës së Skënderbeut, in «Studime Historike», No. 4, pp. 3-22, Tiranë: Instituti i Historisë dhe Gjuhësisë pranë Universitetit Shtetëror të Tiranës.

Buda, A. (2000), Shkrime historike, vol. IV, Tiranë: Toena.

Frashëri, K. \& Islami, S. (1967), Historia e Shqipërisë, vol. I, $2^{\circ}$ ed., Tiranë: Instituti i Historisë dhe Gjuhësisë pranë Universitetit Shtetëror të Tiranës.

Frashëri, K. (1977), Mbi kanunin e Skënderbeut, in «Studime Historike», No. 2, pp. 125-138, Tiranë: Instituti i Historisë pranë Akademisë së Shkencave të Shqipërisë.

Frashëri, K. (2002), Skënderbeu. Jeta dhe vepra, Tiranë: Toena.

Hadri, F. (1985), Shteti feudal centralist shqiptar në epokën e Skënderbeut dhe marrëdhëniet e tij ndërkombëtare me shtetet e tjera, in "Gjurmime Albanologjike», Seria e Shkencave Historike No. XIV (1984), pp. 55-73, Prishtinë: Instituti Albanologjik.

Hahn, G., J. (1854), Albanesische Studien, vol. I, Jena: Friedrich Mauke.

Luarasi, A. (1982), E drejta në shtetin e Skënderbeut, in «Drejtësia popullore», No. 1, pp. 72-88.

Luarasi, A. (1982), Vështrim politiko-juridik mbi disa traktate të Skënderbeut, in «Studime Historike», No. 1, pp. 173-188, Tiranë: Instituti i Historisë pranë Akademisë së Shkencave të Shqipërisë.

Luarasi, A. (1998), Shteti dhe e drejta shqiptare në epokën e Skënderbeut, Tiranë: Luarasi.

Mury, G. (1971), Albania terra dell'uomo nuovo, Milano: Mazzotta.

Noli, F. (1921), Historia e Skenderbeut (Gjerq Kastriotit) Mbretit te Shqiperise 1412-1468, Boston: Shoqëria Arësimi.

Noli, F. (1989), Vepra, vol. IV, Tiranë: Instituti i Historisë pranë Akademisë së Shkencave të Shqipërisë.

Noli, F. (2004), Historia e Skënderbeut. Kryezotit të Arbërisë (1405-1468), Tiranë: Uegen.

Plasari, A. (2010), Skënderbeu. Një histori politike, Tiranë: Instituti shqiptar i studimeve "Gjergj Fishta".

Pulaha, S. (1970), Mbi gjendjen në vitet 20-30 të shekullit XV në tokat shqiptare dhe mbi qëndrimin e klasës feudale kundrejt pushtimit osman, in «Studime Historike», No. 4, pp. 111-157, Tiranë: Instituti i Historisë dhe Gjuhësisë pranë Universitetit Shtetëror të Tiranës.

Sergi, G. (2005), L'idea di medioevo. Fra storia e senso comune, Roma: Donzelli.

Smith, A. (2000), Nazioni e nazionalismo nell'era globale, Trieste: Asterios.

Wickham, C. (2000), Le forme del feudalesimo, in Feudalesimo nell'Alto Medioevo, Spoleto: Centro italiano di studi sull'Alto Medioevo, pp. 16-46.

Wickham, C. (2007), Marxist history-writing for the twenty-first century, Oxford: Oxford University Press. 